Research Paper

\title{
Verteporfin inhibits YAP-induced bladder cancer cell growth and invasion via Hippo signaling pathway
}

\author{
Liang Dong 1,2,\#,凹 , Fan Lin 1,3,\#, Wanjun Wu 1, Yuchen Liu 1, Weiren Huang 1,凹 \\ 1. State Engineering Laboratory of Medical Key Technologies Application of Synthetic Biology, Shenzhen Second People's Hospital, The First Affiliated \\ Hospital of Shenzhen University, Health Science Center of Shenzhen University, Shenzhen 518039, PR China; \\ 2. Department of Biomedical Sciences, City University of Hong Kong, Hong Kong; \\ 3. Guangdong Provincial Key Laboratory of Marine Biotechnology, Shantou University, Shantou 515063, PR China. \\ \#These authors contributed equally to this work \\ $\bowtie$ Corresponding authors: Dr. Liang Dong Email: liandong@cityu.edu.hk and Dr. Weiren Huang Email: pony8980@163.com \\ (c) Ivyspring International Publisher. This is an open access article distributed under the terms of the Creative Commons Attribution (CC BY-NC) license \\ (https://creativecommons.org/licenses/by-nc/4.0/). See http://ivyspring.com/terms for full terms and conditions.
}

Received: 2017.10.23; Accepted: 2018.03.02; Published: 2018.04.03

\begin{abstract}
The highly conserved Hippo signaling pathway is one of the most important pathways involved in tumorigenesis and progress. Previous studies show that YAP, the transcriptional coactivator of Hippo pathway, is expressed highly in many clinical bladder cancer tissues and plays crucial role on bladder cancer progress. To find the YAP-specific target drug and its molecular mechanism in bladder cancer, we apply Verteporfin (VP), a YAP specific inhibitor to function as anti-bladder cancer drug and discover that VP is able to inhibit bladder cancer cell growth and invasion in a dosage dependent manner. Moreover, we demonstrate that VP may inhibit bladder cancer cell growth and invasion via repressing target genes' expression of the Hippo signaling pathway. In further study, we provide evidence that VP is able to inhibit excessive YAP induced bladder cancer cell growth and invasion. To address the repressive function of VP against YAP in bladder cancer, we check the target genes' expression and find VP can dramatically repress YAP overexpression induced Hippo pathway target genes' expression. Taken together, we discover that VP inhibits YAP-induced bladder cancer cell growth and invasion via repressing the target genes' expression of Hippo signaling pathway.
\end{abstract}

Key words: Verteporfin; YAP; bladder cancer; cell growth and invasion

\section{Introduction}

Bladder cancer is a common urological malignant tumor, whose incidence is increasing year by year worldwide. In China, the incidence and mortality rate of bladder cancer are the highest among the urogenital carcinomas, and in the United States, it has the fifth highest incidence rate. However, the molecular mechanism of bladder cancer is far from clear, and there is no effective therapeutic target at present $[1,2]$.Therefore, it is necessary to study the mechanism of bladder cancer formation and screen the effective target drug for bladder cancer treatment.

Many signaling pathways that affect the survival of bladder cancer cell have been reported, such as MAPK, JAK-STAT, NF-kB, mTOR, et al [2]. Most recently, evolutionarily conserved Hippo pathway has been discovered to play an important role during tumorigenesis and progression of bladder cancer [3]. The Hippo signaling pathway functions via the transcriptional coactivator Yes-associated protein (YAP) to regulate cell growth and migration in mammals $[4,5]$. YAP is a potential oncogene which is upregulated in various tumors. Previous reports showed YAP was expressed highly in bladder cancer clinical samples and the expression level of YAP was crucial for cell growth and migration in bladder cancer [6-11]. These studies indicated that YAP may become a promising drug target for bladder cancer treatment.

According to preceding investigation, Verteporfin (VP), a YAP specific inhibitor, can block 
the interaction between transcriptional coactivator YAP and transcriptional factor TEAD to repress YAP's function [12].During the past few years, several researchers discovered that $\mathrm{VP}$ is able to restrain cancer cell growth in some tumors, including retinoblastoma, endometrial and ovarian cancers [13-15]. However, the function and mechanism of VP in bladder cancer were not yet addressed. Therefore, it is possible that VP may inhibit bladder cancer cell growth through suppressing $\mathrm{YAP}^{\prime}$ s activity.

In this study, we proved that Verteporfin inhibited bladder cancer cell growth and invasion in a dosage dependent manner. Moreover, we found that VP suppressed the target genes' expression of the Hippo signaling pathway to restrict bladder cancer cell growth and invasion. Our further study provided evidence that VP was able to dramatically repress bladder cancer cell growth and invasion caused by YAP overexpression. Finally, to address the mechanism that VP suppresses YAP to inhibit bladder cancer progress, we checked the target genes' expression of Hippo signaling pathway and found that VP was able to obviously repress excessive YAP induced Hippo pathway target genes' expression. Taking together, we find that VP inhibits YAP-induced bladder cancer cell growth and invasion via repressing the target genes' expression of Hippo signaling pathway. These results would provide a clue to develop VP as a YAP specific target drug to intervene bladder cancer, especially for YAP highly expressed cases.

\section{Materials and Methods}

\section{Plasmids and reagent}

The YAP plasmid was bought from Addgene (ID NO: 42555) (http://www.addgene.org/). Verteporfin was purchased from Sigma (USA)

\section{Verteporfin (VP) treatment}

Verteporfin (Sigma, USA) was dissolved in DMSO and added to the medium for a final concentration of $2 \mu \mathrm{g} / \mathrm{ml}$ or $10 \mu \mathrm{g} / \mathrm{ml}$ in indicated experiments. Equal concentration of DMSO was added in the control cells.

\section{Cell culture and transfections}

Human embryonic kidney cell line (HEK 293T) and bladder cancer cell lines (5637 and UMUC-3) were purchased from the Institute of Cell Biology, Chinese Academy of Sciences, Shanghai, China. The 293T cell was cultured in DMEM media. The 5637 and UMUC-3 cells were respectively maintained in RPMI-1640 media or DMEM media. Both RPMI-1640 media and DMEM media were supplemented with $10 \%$ FBS and $1 \%$ antibiotics $(100 \mathrm{U} / \mathrm{ml}$ penicillin and
$100 \mathrm{\mu g} / \mathrm{ml}$ streptomycin sulfates). All cells were cultured at $37^{\circ} \mathrm{C}$ in an atmosphere of $5 \% \mathrm{CO}_{2}$.

In all the cell transfection processes, the corresponding cells were transfected with Lipofactamine 3000 (Invitrogen) following the manufacture's instruction.

\section{Quantitative Real-Time PCR Analysis}

Total RNAs from corresponding cells were extracted and isolated using the Trizol reagent (Invitrogen, USA) following the manufacture's protocol. And then cDNAs were synthesized using SuperScript III ${ }^{\circledR}$ kit (Invitrogen, USA). Quantitative PCR (qPCR) was done on the ABI PRISM 7000 Fluorescent Quantitative PCR system (Applied Biosystems, USA) by using SYBR Green Premix (Takara, Japan). All the indicated samples were normalized to gapdh and then the relative mRNA levels were calculated via using $\triangle \triangle \mathrm{Ct}$ way. The primers were shown as below in $5^{\prime}$ to $3^{\prime}$ direction:

gapdh F: TCATCCCTGCCTCTACTG; gapdh R: TGCTTCACCACCTTCTTG;

CTGF F: CCAATGACAACGCCTCCTG;

CTGF R: TGGTGCAGCCAGAAAGCTC;

cyr61 F: AGCCTCGCATCCTATACAACC;

cyr61 R: TTCTTTCACAAGGCGGCACTC;

ANKRD1 F: CACTTCTAGCCCACCCTGTGA;

ANKRD1 R: CCACAGGTTCCGTAATGATTT.

\section{CCK-8 assay}

The effects of VP and YAP on cell growth were determined by Cell Counting Kit- 8 assay (Transgene, China). In brief, $5 \times 10^{3}$ cells per well were seeded in a 96-well plate for $12 \mathrm{~h}$ culture and then transfected or treated with corresponding plasmids and/or VP (10 $\mu \mathrm{g} / \mathrm{ml}$ ) as mentioned previously. After transfection for $48 \mathrm{~h}, 100 \mathrm{\mu l}$ fresh medium with $10 \%$ of CCK-8 was replaced into each well and the cells were cultured for another one hour. The absorbance of $450 \mathrm{~nm}$ was detected by using an ELISA microplate reader (Bio-Rad, Hercules, CA, USA). Every experiment was repeated for three times.

\section{Edu staining assay}

The effects of YAP and VP on cell proliferation were determined by Ethynyl-2-deoxyuridine incorporation assay by using Cell-Light ${ }^{\mathrm{TM}}$ EdU Apollo®567 In Vitro Imaging Kit (Ribobio, China). In brief, after transfection or VP treatment for $48 \mathrm{~h}$, the Edu was added into every well in a finial concentration of $50 \mu \mathrm{M}$. After two hours' culture, cells were fixed with $4 \%$ paraformaldehyde in PBS at room temperature. After three times' washing in PBST (PBS containing $0.1 \%$ Triton X-100), cells were incubated with $1 \times$ Apollo solution for half an hour at room 
temperature (RT) in the dark. Finally, cells were subjected to $1 \mathrm{x}$ Hoechst nuclear dye staining for 30 min and then detected by fluorescence microscopy.

\section{Transwell assay}

Transwell assays were carried out using 24-well BioCoat cell culture inserts (BD Biosciences). The upper surface of 6.4-mm diameter filters with $8 \mu \mathrm{m}$ pores were precoated with extracellular matrix coating (Matrigel). After treatment with either DMSO or VP $(2 \mu \mathrm{g} / \mathrm{ml}$ or $10 \mu \mathrm{g} / \mathrm{ml}, 24 \mathrm{~h})$, cells were washed twice with sterile $1 x$ PBS to remove the dead cells, and then harvested and counted using Countess II FL counter (Life Technology). 10,000 viable cells in 1\% serum medium were seeded on to the upper chamber of each insert. And then complete medium was added to the bottom chamber. Following $24 \mathrm{~h}$ of incubation, invasive cells on the lower surface of the filters were fixed and stained with the $1 \%$ crystal violet, and counted.

\section{Statistical analysis}

Every experiment was performed in triplicate and data were presented as mean \pm standard deviation (SD). Statistical analysis was conducted by Student's t-test or ANOVA using SPSS version 19.0 software (SPSS Inc. Chicago, IL, USA). $p<0.05$ was considered to be statistically significant.

\section{Results}

\section{Verteporfin inhibits bladder cancer cell growth in a dosage dependent manner}

To determine whether Verteporfin (VP) inhibits human bladder cancer cell growth, we checked its effect on the growth of human bladder cancer cell lines, 5637 and UMUC-3.The molecular structure of $\mathrm{VP}$ was revealed in Figure 1A. The cell lines were treated by DMSO, $2 \mu \mathrm{g} / \mathrm{ml} \mathrm{VP}$ or $10 \mu \mathrm{g} / \mathrm{ml} \mathrm{VP}$ as shown in Figure 1. 5637 bladder cancer cell line treated by VP demonstrated dosage-dependent decrease in cell growth measured by cell number count assay (CCK-8) (Figure 1B). Consistently, a significant inhibition effect on cell proliferation was observed in VP treated 5637 cells using the Edu staining assay (Figure 1C-1 $\mathrm{E}^{\prime \prime}$ ). To confirm the cell growth inhibition function of VP, we treated another bladder cancer cell line UMUC-3 with VP, and set up CCK-8 and Edu staining assay. Very similarly to 5637 cell line, the growth and proliferation of the UMUC-3 cells were dramatically repressed by VP in a dosage dependent manner (Figure $\left.1 \mathrm{~F}-\mathrm{I}^{\prime \prime}\right)$. Taken together, these data suggested that VP inhibited bladder cancer cell growth in a dosage dependent manner.

\section{VP inhibits bladder cancer cell invasion in a dosage dependent manner}

As YAP played crucial role on cancer cell invasion, we also checked the effect of VP on bladder cancer cell invasion. In 5637 cells, we discovered that VP treatment obviously suppressed the cell invasion ability in a dosage dependent manner (Figure 2A-2C). Similarly, UMUC-3 cell invasion activity was clearly inhibited by $\mathrm{VP}$ in a dosage dependent manner (Figure 2D-2F). In all, these results demonstrated that the bladder cancer cell invasion ability was dramatically inhibited by VP.

\section{VP represses the target genes' expression of Hippo signaling pathway}

To investigate the potential mechanism that VP suppressed the bladder cancer cell growth and invasion, we detected the expression of the target genes in this pathway (such as CTGF, cyr61 and ANKRD1) by qPCR assay, which play important role in cell growth and invasion. It was shown that VP visibly repressed the target genes' expression in a dosage dependent manner in 293T cells (Figure 3A-C). In order to verify the inhibition effect of the target genes in bladder cancer cell line, we set up qPCR assay and found VP obviously downregulated the target genes' expression in 5637 cells (Figure S1A-C). In summary, these data indicated that VP may repress the target genes' expression of Hippo signaling pathway to inhibit bladder cancer progress.

\section{VP inhibits YAP induced bladder cancer cell growth and invasion}

To gain insight into the $\mathrm{VP}^{\prime} \mathrm{s}$ effect on YAP during bladder cancer tumorigenesis and development, we overexpressed YAP in 5637 cell to mimic YAP highly expressed bladder cancer cases, and performed CCK-8 assay to check the cell growth effect. It was shown that more cells were detected by this assay, when YAP was overexpressed (Figure 4A). Very interestingly, VP was able to efficiently suppress YAP overexpression induced bladder cancer cell growth (Figure 4A). To further confirm this finding, we performed the Edu staining assay to check the cell proliferation effect of YAP and VP. Consistently, excessive bladder cancer cell proliferation caused by overexpression of YAP can be obviously blocked by VP treatment (Figure 4B-4E"). Furthermore, YAP overexpression significantly promoted 5637 cell invasion by transwell assay (Figure $4 \mathrm{~F}-4 \mathrm{G}$ ) and the promotion effect was able to be restrained by VP (Figure 4F-4I). To verify the inhibition effect of VP against YAP, we set up Edu staining assay in UMUC-3 cell and discovered that VP similarly repressed the cell proliferation caused by excessive 
YAP (Figure S2A-D"). Taken together, these data suggested that bladder cancer cell growth and invasion promotion effect induced by YAP overexpression can be dramatically inhibited by VP treatment.

\section{VP is sufficient to downregulate YAP induced target genes' expression of Hippo signaling pathway}

To investigate the potential mechanism of the bladder cancer cell growth and invasion inhibition effect of VP against YAP, we tested the Hippo signaling pathway target genes' expression via qPCR assay in $293 \mathrm{~T}$ cells. As a result, we demonstrated that
YAP upregulated the target genes' expression while VP downregulated it and VP was sufficient to strikingly repress YAP overexpression induced target genes' expression of Hippo signaling pathway (CTGF, cyr61 and ANKRD1) (Figure 5A-5C). To ensure if this kind of effect is consistent in bladder cancer cells, we performed the same assay in bladder cancer 5637 cell and uncovered similar function of VP against YAP (Figure S3A-C). Collectively, these results indicated that VP may inhibit YAP induced bladder cancer progress via restricting Hippo pathway target genes' expression.
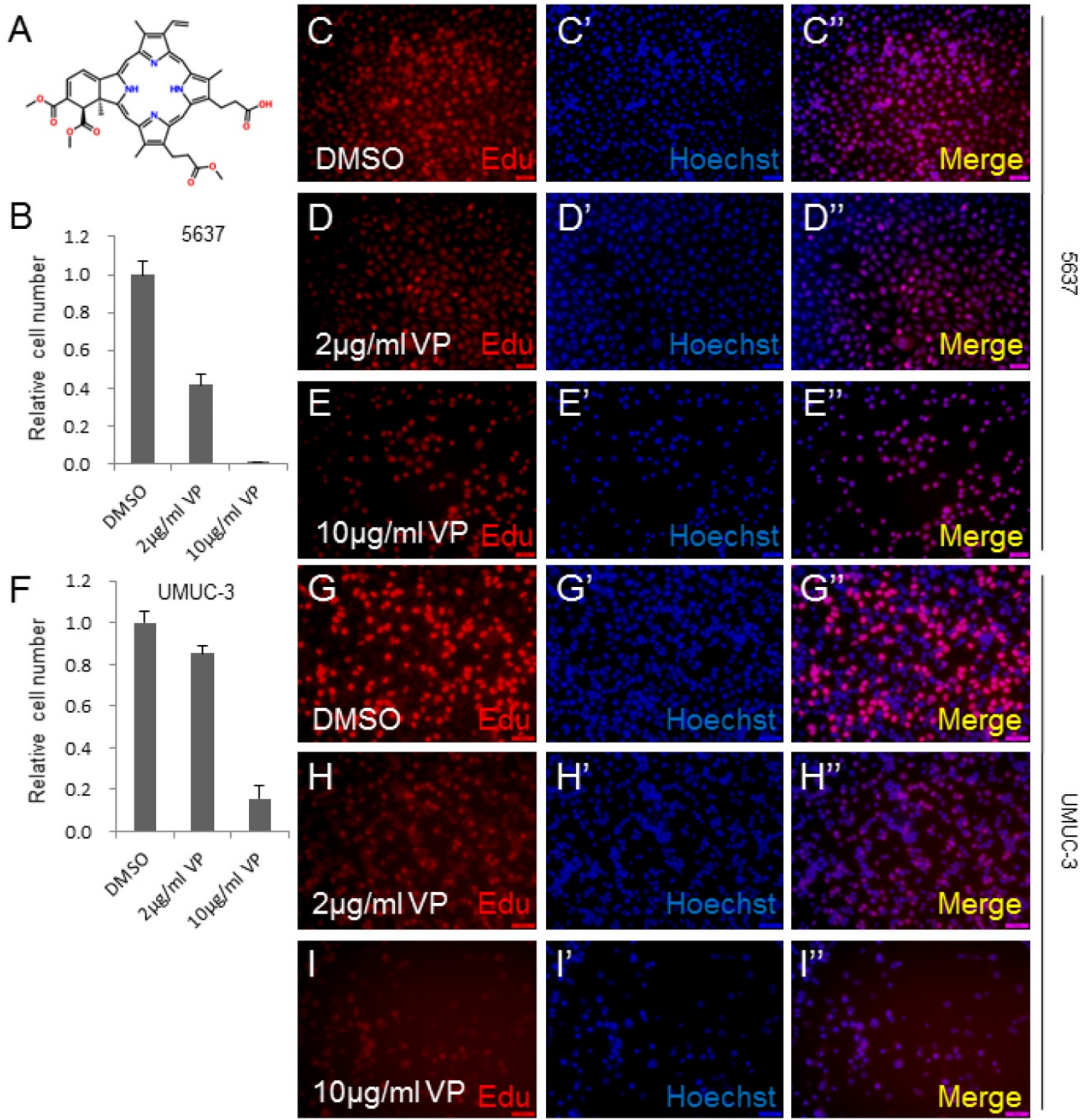

Figure 1. VP inhibits bladder cancer cell growth in a dosage dependent manner. (A) The chemical structure of Verteporfin (VP); (B) 5637 cell growth treated by DMSO, $2 \mu \mathrm{g} / \mathrm{ml} \mathrm{VP} \mathrm{or} 10 \mu \mathrm{g} / \mathrm{ml} \mathrm{VP}$ is measured by CCK-8 assay; (C-E) 5637 cell proliferation treated by DMSO, $2 \mu \mathrm{g} / \mathrm{ml}$ VP or $10 \mu \mathrm{g} / \mathrm{ml}$ VP is measured by Edu staining assay; (F) UMUC-3 cell growth treated by DMSO, $2 \mu \mathrm{g} / \mathrm{ml} \mathrm{VP} \mathrm{or} 10 \mu \mathrm{g} / \mathrm{ml} \mathrm{VP}$ is measured by CCK-8 assay; (G-I) UMUC-3 cell proliferation treated by DMSO, $2 \mu \mathrm{g} / \mathrm{ml} \mathrm{VP} \mathrm{or} 10 \mu \mathrm{g} / \mathrm{ml} \mathrm{VP}$ is measured by Edu staining assay. 


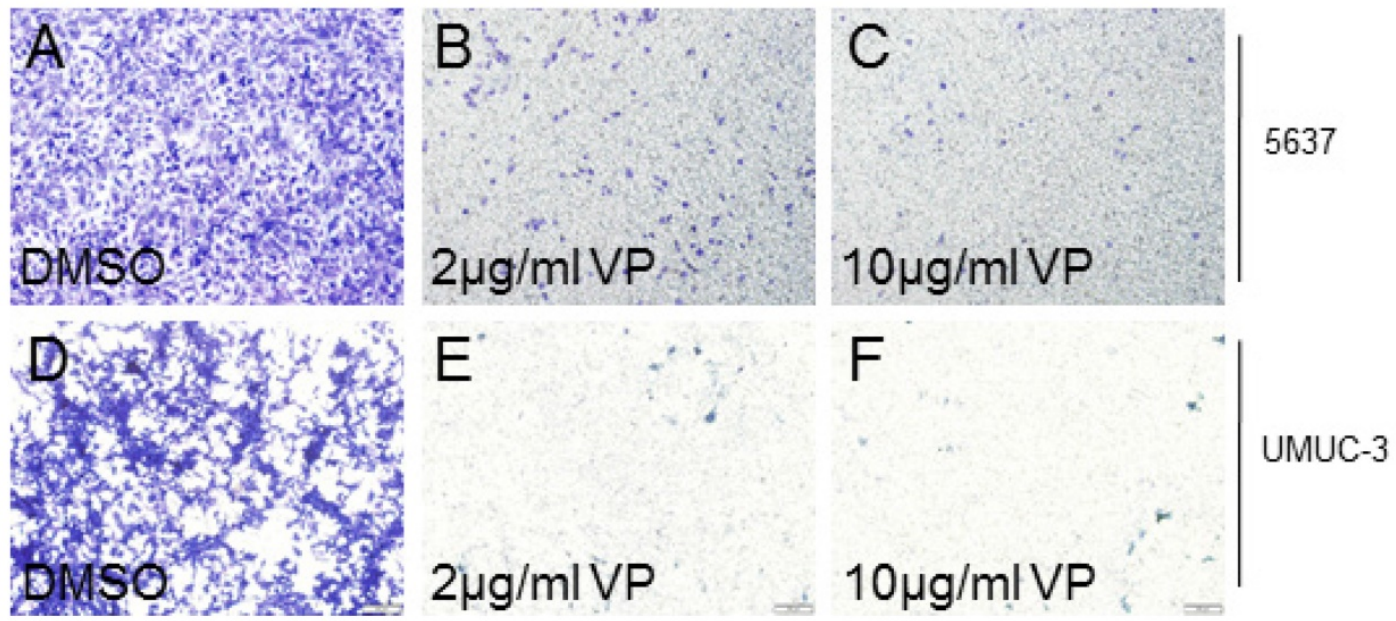

Figure 2. VP inhibits bladder cancer cell invasion in a dosage dependent manner. (A-C) 5637 cell invasion treated by DMSO, $2 \mu g / \mathrm{ml} \mathrm{VP} \mathrm{or} 10 \mu g / \mathrm{ml} \mathrm{VP}$ is measured by transwell assay; (D-F) UMUC-3 cell invasion treated by DMSO, $2 \mu \mathrm{g} / \mathrm{ml} \mathrm{VP} \mathrm{or} 10 \mu \mathrm{g} / \mathrm{ml} \mathrm{VP} \mathrm{is} \mathrm{measured} \mathrm{by} \mathrm{transwell} \mathrm{assay.}$
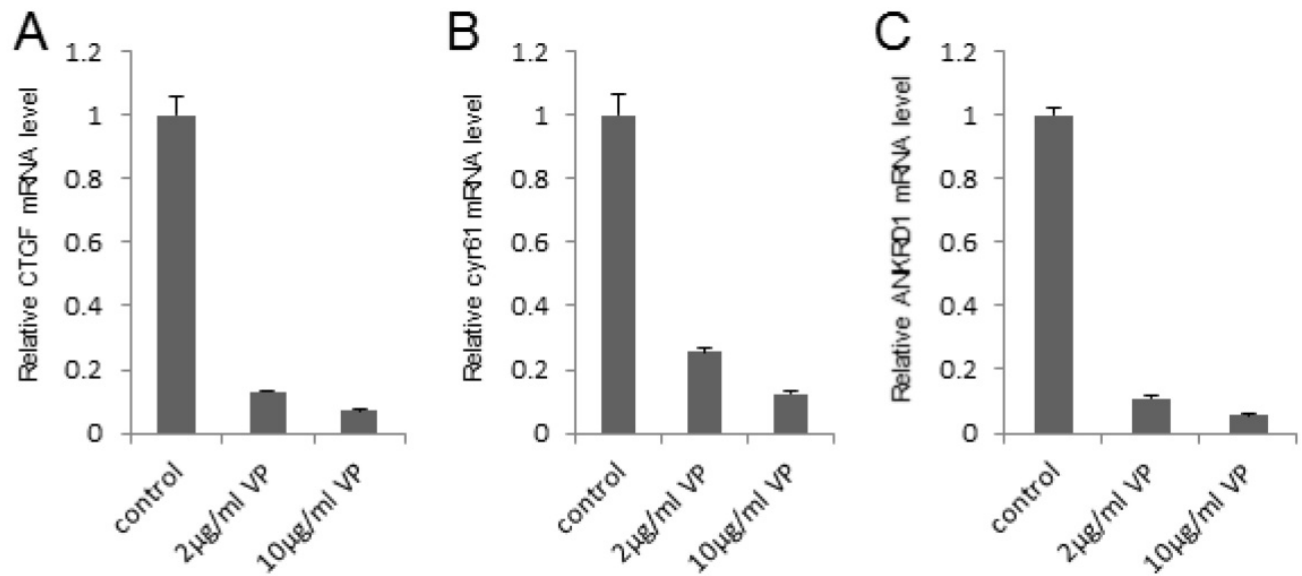

Figure 3. VP represses the target genes' expression of Hippo signaling pathway in a dosage dependent manner. (A-C) qPCR to check the expression level of target genes (CTGF, cyr6I and ANKRDI) of the Hippo pathway in 293T cells and each sample was repeated three times for qPCR assay. 293T cells were treated by DMSO, $2 \mu \mathrm{g} / \mathrm{ml} \mathrm{VP} \mathrm{or} 10 \mu \mathrm{g} / \mathrm{ml} \mathrm{VP}$, respectively.

Taking together, we proposed an underlying working model that Verteporfin inhibits YAP-induced bladder cancer cell growth and invasion via Hippo signaling pathway, which provided a potential novel YAP-targeted drug for bladder cancer therapy (Figure 6). In this working model, YAP is highly expressed in some bladder cancer cells and promotes bladder cancer cell progress, while VP, as specific inhibitor of YAP, is able to abolish YAP induced bladder cancer cell growth and invasion. This kind of bladder cancer cell progress inhibition effect of VP against YAP may be due to $\mathrm{VP}^{\prime} \mathrm{s}$ repressive function on Hippo pathway's target genes' expression (Figure 6).

\section{Discussion}

This study demonstrated that Verteporfin (VP) is able to inhibit bladder cancer cell growth and invasion in a dosage dependent manner. Moreover, VP may inhibit bladder cancer cell growth and invasion via repression target genes' expression of the Hippo signaling pathway. Furthermore, VP treatment is able to inhibit excessive YAP induced bladder cancer cell growth and invasion. Besides, VP can clearly downregulate YAP overexpression induced Hippo pathway target genes' expression. Collectively, we uncovered that VP downregulated the target genes' expression of Hippo signaling pathway to suppress YAP-induced bladder cancer cell growth and invasion. VP may become a latent effective YAP-targeted drug for bladder cancer therapy.

Our discovery had demonstrated that VP is able to repress YAP activity to inhibit bladder cancer cell growth and invasion. Our findings were consistent with others' report in some other kinds of tumors [12-19]. Therefore, the research about VP may pave an alternative way for the design of anti-cancer drugs. 


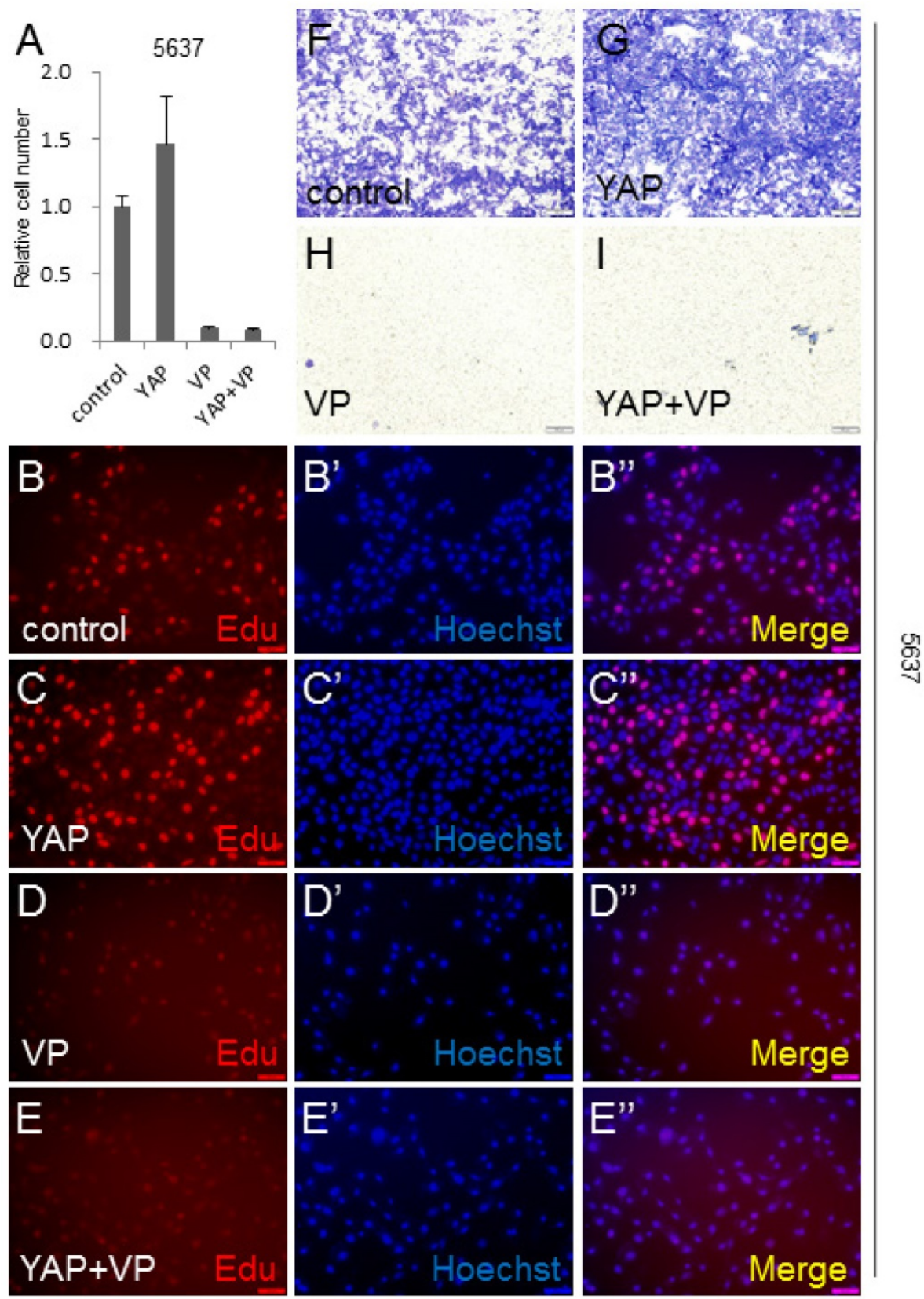

Figure 4. VP inhibits YAP induced bladder cancer cell growth and invasion. (A) 5637 cell growth after being transfected or/and treated by YAP or/and VP $(10 \mathrm{\mu g} / \mathrm{ml})$ is measured by CCK-8 assay; (B-E) 5637 cell proliferation after being transfected or/and treated by YAP or/and VP is measured by Edu staining assay; (F-l) 5637 cell invasion after being transfected or/and treated by YAP or/and VP is measured by transwell assay.
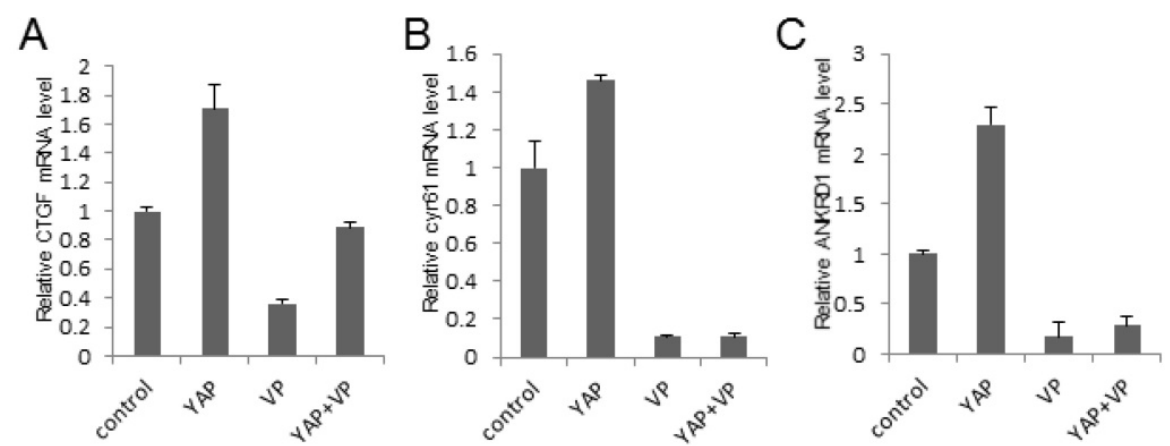

Figure 5. VP inhibits YAP induced Hippo pathway target genes' expression. (A-C) qPCR to check the expression level of target genes (CTGF, cyr6I and ANKRDI) of the Hippo pathway in 293T cells and each sample was repeated three times for QPCR assay. 293T cells were transfected or/and treated by YAP or/and VP $(10 \mu \mathrm{g} / \mathrm{ml})$ as showing in the figures. 


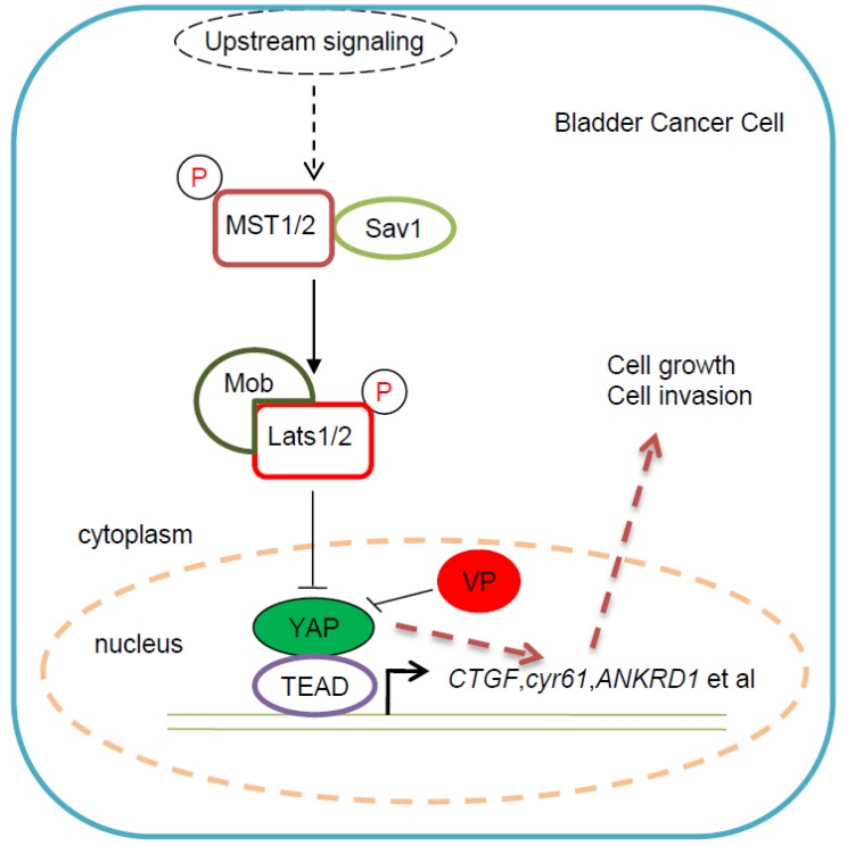

Figure 6. Proposed working model for VP in bladder cancer cell. As previous description, YAP was highly expressed in some bladder cancer patients and played dominant role in bladder cancer progress. However, there is still no efficient YAP-targeted drug for bladder cancer treatment. According to our study on VP, we proposed the working model: VP suppressed bladder cancer cell growth and invasion via specifically repressing YAP activity; As YAP specific inhibitor of YAP, VP was able to efficiently downregulate Hippo pathway's target genes' expression and then restricted bladder cancer cell growth and invasion; VP might become a potential YAP-targeted drug for bladder cancer treatment, especially for YAP highly expressed cases.

However, VP as an effective YAP-targeted drug, is inevitable for a certain toxic side effect to normal cells. Hence, it is necessary to modify VP to reduce its toxic effect and enhance its anti-cancer efficiency. As VP is not used in clinical treatment for all kinds of tumors, there is a long way to develop VP as an anti-cancer chemical drug. It is necessary to compare the effect of VP with well-known chemical anti-cancer drug, such as sorafenib etc. The future work will be to search for high efficiency, low toxicity and novel YAP-targeted anti-tumor drugs.

\section{Conclusions}

Verteporfin (VP) is able to inhibit bladder cancer cell growth and invasion in a dosage dependent manner. VP may repress target genes' expression of the Hippo signaling pathway to inhibit bladder cancer cell growth and invasion. VP is able to inhibit YAP overexpression induced bladder cancer cell growth and invasion via repressing an excess of YAP induced Hippo pathway target genes' expression. VP functions as a YAP specific inhibitor to intervene bladder cancer progress.

\section{Abbreviations}

VP: Verteporfin; YAP: Yes-associated protein; TEAD: TEA domain transcription factor; CTGF: connective tissue growth factor; Cyr61: Cysteine-rich angiogenic inducer 61; ANKRD1: Ankyrin Repeat Domain 1.

\section{Supplementary Material}

Supplementary figures.

http://www.medsci.org/v15p0645s1.pdf

\section{Acknowledgements}

This work was supported by National Natural Science Foundation of China (81702510, 81702764), the Planned Science and Technology Project of Guang dong Province, China (2017A020215004, 2017A020215 120), China Postdoctoral Foundation (2015M582462), Shenzhen Municipal Government of China (ZDSYS2 01504301722174, JCYJ20150330102720130, GJHZ20150 316154912494, JCYJ20160425100840929, JCYJ2017030 6091121656), Special Support Funds of Shenzhen for Introduced High-Level Medical Team, and Shenzhen High-Level Medical Discipline Development Program (2016031638).

\section{Competing Interests}

The authors have declared that no competing interest exists.

\section{References}

1. Rouanne M, Loriot $\mathrm{Y}$, Lebret $\mathrm{T}$, Soria JC. Novel therapeutic targets in advanced urothelial carcinoma. Critical reviews in oncology/hematology. 2015.

2. Abbosh PH, McConkey DJ, Plimack ER. Targeting Signaling Transduction Pathways in Bladder Cancer. Current oncology reports. 2015; 17: 58.

3. Xia J, Zeng M, Zhu H, Chen X, Weng Z, Li S. Emerging role of Hippo signalling pathway in bladder cancer. Journal of cellular and molecular medicine. 2017.

4. Mo JS, Park HW, Guan KL. The Hippo signaling pathway in stem cell biology and cancer. EMBO reports. 2014; 15: 642-56.

5. Dong L, Li J, Huang H, Yin MX, Xu J, Li P, et al. Growth suppressor lingerer regulates bantam microRNA to restrict organ size. Journal of molecular cell biology. 2015.

6. Li S, Yu Z, Chen SS, Li F, Lei CY, Chen XX, et al. The YAP1 oncogene contributes to bladder cancer cell proliferation and migration by regulating the H19 long noncoding RNA. Urologic oncology. 2015.

7. Ciamporcero E, Shen H, Ramakrishnan S, Yu Ku S, Chintala S, Shen L, et al. YAP activation protects urothelial cell carcinoma from treatment-induced DNA damage. Oncogene. 2015.

8. Gao Y, Shi Q, Xu S, Du C, Liang L, Wu K, et al. Curcumin promotes KLF5 proteasome degradation through downregulating YAP/TAZ in bladder cancer cells. International journal of molecular sciences. 2014; 15: 15173-87.

9. Liu JY, Li YH, Lin HX, Liao YJ, Mai SJ, Liu ZW, et al. Overexpression of YAP1 contributes to progressive features and poor prognosis of human urothelial carcinoma of the bladder. BMC cancer. 2013; 13: 349.

10. Zhang L, Ren F, Zhang Q, Chen Y, Wang B, Jiang J. The TEAD/TEF family of transcription factor Scalloped mediates Hippo signaling in organ size control. Developmental cell. 2008; 14: 377-87

11. Dong L, Lin F, Wu W, Huang W, Cai Z. Transcriptional cofactor Mask2 is required for YAP-induced cell growth and migration in bladder cancer cell. Journal of Cancer. 2016; 7: 2132-8.

12. Brodowska K, Al-Moujahed A, Marmalidou A, Meyer Zu Horste M, Cichy J, Miller JW, et al. The clinically used photosensitizer Verteporfin (VP) inhibits YAP-TEAD and human retinoblastoma cell growth in vitro without light activation. Experimental eye research. 2014; 124: 67-73. 
13. Dasari VR, Mazack V, Feng W, Nash J, Carey DJ, Gogoi R. Verteporfin exhibits YAP-independent anti-proliferative and cytotoxic effects in endometrial cancer cells. Oncotarget. 2017; 8: 28628-40.

14. Ma YW, Liu YZ, Pan JX. Verteporfin induces apoptosis and eliminates cancer stem-like cells in uveal melanoma in the absence of light activation. American journal of cancer research. 2016; 6: 2816-30.

15. Feng J, Gou J, Jia J, Yi T, Cui T, Li Z. Verteporfin, a suppressor of YAP-TEAD complex, presents promising antitumor properties on ovarian cancer. OncoTargets and therapy. 2016; 9: 5371-81

16. Chen M, Zhong L, Yao SF, Zhao Y, Liu L, Li LW, et al. Verteporfin Inhibits Cell Proliferation and Induces Apoptosis in Human Leukemia NB4 Cells without Light Activation. International journal of medical sciences. 2017; 14: 1031-9.

17. Al-Moujahed A, Brodowska K, Stryjewski TP, Efstathiou NE, Vasilikos I, Cichy J, et al. Verteporfin inhibits growth of human glioma in vitro without light activation. Scientific reports. 2017; 7: 7602

18. Celli JP, Solban N, Liang A, Pereira SP, Hasan T. Verteporfin-based photodynamic therapy overcomes gemcitabine insensitivity in a panel of pancreatic cancer cell lines. Lasers in surgery and medicine. 2011; 43: 565-74.

19. Hu Z, Rao B, Chen S, Duanmu J. Targeting tissue factor on tumour cells and angiogenic vascular endothelial cells by factor VII-targeted verteporfin photodynamic therapy for breast cancer in vitro and in vivo in mice. BMC cancer. 2010; 10: 235. 